\title{
On colorings avoiding a rainbow cycle and a fixed monochromatic subgraph
}

\author{
Maria Axenovich* \\ JiHyeok Choi \\ Department of Mathematics, Iowa State University, Ames, IA 50011 \\ axenovic@iastate.edu, jchoi@iastate.edu
}

Submitted: Apr 23, 2009; Accepted: Feb 7, 2010; Published: Feb 22, 2010

Mathematics Subject Classification: 05C15, 05C55

\begin{abstract}
Let $H$ and $G$ be two graphs on fixed number of vertices. An edge coloring of a complete graph is called $(H, G)$-good if there is no monochromatic copy of $G$ and no rainbow (totally multicolored) copy of $H$ in this coloring. As shown by Jamison and West, an $(H, G)$-good coloring of an arbitrarily large complete graph exists unless either $G$ is a star or $H$ is a forest. The largest number of colors in an $(H, G)$-good coloring of $K_{n}$ is denoted $\max R(n, G, H)$. For graphs $H$ which can not be vertexpartitioned into at most two induced forests, $\max R(n, G, H)$ has been determined asymptotically. Determining $\max R(n ; G, H)$ is challenging for other graphs $H$, in particular for bipartite graphs or even for cycles. This manuscript treats the case when $H$ is a cycle. The value of $\max R\left(n, G, C_{k}\right)$ is determined for all graphs $G$ whose edges do not induce a star.
\end{abstract}

\section{Introduction and main results}

For two graphs $G$ and $H$, an edge coloring of a complete graph is called $(H, G)$-good if there is no monochromatic copy of $G$ and no rainbow (totally multicolored) copy of $H$ in this coloring. The mixed anti-Ramsey numbers, $\max R(n ; G, H), \min R(n ; G, H)$ are the maximum, minimum number of colors in an $(H, G)$-good coloring of $K_{n}$, respectively. The number $\max R(n ; G, H)$ is closely related to the classical anti-Ramsey number $A R(n, H)$, the largest number of colors in an edge-coloring of $K_{n}$ with no rainbow copy of $H$ introduced by Erdős, Simonovits and Sós [9]. The number $\min R(n ; G, H)$ is closely related to

*The first author's research supported in part by NSA grant H98230-09-1-0063 and NSF grant DMS0901008. 
the classical multicolor Ramsey number $R_{k}(G)$, the largest $n$ such that there is a coloring of edges of $K_{n}$ with $k$ colors and no monochromatic copy of $G$. The mixed Ramsey number $\min R(n ; G, H)$ has been investigated in $[3,13,11]$.

This manuscript addresses $\max R(n ; G, H)$. As shown by Jamison and West [14], an $(H, G)$-good coloring of an arbitrarily large complete graph exists unless either $G$ is a star or $H$ is a forest. Let $a(H)$ be the smallest number of induced forests vertex-partitioning the graph $H$. This parameter is called a vertex arboricity. Axenovich and Iverson [3] proved the following.

Theorem 1. Let $G$ be a graph whose edges do not induce a star and $H$ be a graph with $a(H) \geqslant 3$. Then $\max R(n ; G, H)=\frac{n^{2}}{2}\left(1-\frac{1}{a(H)-1}\right)(1+o(1))$.

When $a(H)=2$, the problem is challenging and only few isolated results are known [3]. Even in the case when $H$ is a cycle, the problem is nontrivial. This manuscript addresses this case. Since $\left(C_{k}, G\right)$-good colorings do not contain rainbow $C_{k}$, it follows that

$$
\max R\left(n ; G, C_{k}\right) \leqslant A R\left(n, C_{k}\right)=n\left(\frac{k-2}{2}+\frac{1}{k-1}\right)+O(1),
$$

where the equality is proven by Montellano-Ballesteros and Neumann-Lara [16]. We show that $\max R\left(n ; G, C_{k}\right)=A R\left(n ; C_{k}\right)$ when $G$ is either bipartite with large enough parts, or a graph with chromatic number at least 3 . In case when $G$ is bipartite with a "small" part, $\max R\left(n ; G, C_{k}\right)$ depends mostly on $G$, namely, on the size of the "small" part. Below is the exact formulation of the main result.

If a graph $G$ is bipartite, we let $s(G)=\min \left\{s: G \subseteq K_{s, r}, s \leqslant r\right.$ for some $\left.r\right\}$ and $t(G)=|V(G)|-s(G)$. I.e., $s(G)$ is the sum of the sizes of smaller parts over all components of $G$.

Theorem 2. Let $k \geqslant 3$ be an integer and $G$ be a graph whose edges do not induce a star. Let $s=s(G)$ and $t=t(G)$ if $G$ is bipartite. There are constants $n_{0}=n_{0}(G, k)$ and $g=g(G, k)$ such that for all $n \geqslant n_{0}$

$$
\max R\left(n ; G, C_{k}\right)=\left\{\begin{array}{l}
n\left(\frac{k-2}{2}+\frac{1}{k-1}\right)+O(1), \quad \text { if }(\chi(G)=2 \text { and } s \geqslant k) \text { or }(\chi(G) \geqslant 3) \\
n\left(\frac{s-2}{2}+\frac{1}{s-1}\right)+g, \quad \text { otherwise }
\end{array}\right.
$$

Here $g=g(G, k)=E R^{2}(s+t, 3 s k+t+1, k)$, where the number ER denotes the ErdősRado number stated in section 2. Note that it is sufficient to take $g(G, k)=2^{c \ell^{2} \log \ell}$, where $\ell=3 s k+t+1$.

We give the definitions and some observations in section 2, the proof of the main theorem in section 3 and some more accurate bounds for the case when $H=C_{4}$ in the last section of the manuscript.

\section{Definitions and preliminary results}

First we shall define a few special edge colorings of a complete graph: lexical, weakly lexical, $k$-anticyclic, $c^{*}$ and $c^{* *}$. 
Let $c: E\left(K_{n}\right) \rightarrow \mathbb{N}$ be an edge coloring of a complete graph on $n$ vertices for some fixed $n$.

We say that $c$ is a weakly lexical coloring if the vertices can be ordered $v_{1}, \ldots, v_{n}$, and the colors can be renamed such that there is a function $\lambda: V\left(K_{n}\right) \rightarrow \mathbb{N}$, and $c\left(v_{i} v_{j}\right)=$ $\lambda\left(v_{\min \{i, j\}}\right)$, for $1 \leqslant i, j \leqslant n$. In particular, if $\lambda$ is one to one, then $c$ is called a lexical coloring.

We say that $c$ is a $k$-anticyclic coloring if there is no rainbow copy of $C_{k}$, and there is a partition of $V\left(K_{n}\right)$ into sets $V_{0}, V_{1}, \ldots, V_{m}$ with $0 \leqslant\left|V_{0}\right|<k-1$ and $\left|V_{1}\right|=\cdots=$ $\left|V_{m}\right|=k-1$, where $m=\left\lfloor\frac{n}{k-1}\right\rfloor$, such that for $i, j$ with $0 \leqslant i<j \leqslant m$, all edges between $V_{i}$ and $V_{j}$ have the same color, and the edges spanned by each $V_{i}, i=0, \ldots, m$ have new distinct colors using pairwise disjoint sets of colors.

We denote a fixed coloring from the set of $k$-anticyclic colorings of $K_{n}$ such that the color of any edges between $V_{i}$ and $V_{j}$ is $\min \{i, j\}$ by $c^{*}$.

Finally, we need one more coloring, $c^{* *}$, of $K_{n}$. Let $c^{* *}$ be a fixed coloring from the set of the following colorings of $E\left(K_{n}\right)$; let the vertex set $V\left(K_{n}\right)$ be a disjoint union of $V_{0}, V_{1}, \ldots, V_{m}$ with $0 \leqslant\left|V_{0}\right|<s-1,\left|V_{1}\right|=\cdots=\left|V_{m-1}\right|=s-1$, and $\left|V_{m}\right|=k-1$, where $m-1=\left\lfloor\frac{n-k+1}{s-1}\right\rfloor$. Let the color of each edge between $V_{i}$ and $V_{j}$ for $0 \leqslant i<j \leqslant m$ be $i$. Color the edges spanned by each $V_{i}, i=0, \ldots, m$ with new distinct colors using pairwise disjoint sets of colors.

For a coloring $c$, let the number of colors used by $c$ be denoted by $|c|$. Observe that $c^{*}$ is a blow-up of a lexical coloring with parts inducing rainbow complete subgraphs. Any monochromatic bipartite subgraph in $c^{*}$ and $c^{* *}$ is a subgraph of $K_{k-1, t}$ and $K_{s-1, t}$ for some $t$, respectively. Also we easily see that if $c$ is $k$-anticyclic, then

$$
\begin{gathered}
|c| \leqslant\left|c^{*}\right|=n\left(\frac{k-2}{2}+\frac{1}{k-1}\right)+O(1), \\
\left|c^{* *}\right|=n\left(\frac{s-2}{2}+\frac{1}{s-1}\right)+O(1) .
\end{gathered}
$$

Let $K=K_{n}$. For disjoint sets $X, Y \subseteq V$, let $K[X]$ be the subgraph of $K$ induced by $X$, and let $K[X, Y]$ be the bipartite subgraph of $K$ induced by $X$ and $Y$. Let $c(X)$ and $c(X, Y)$ denote the sets of colors used in $K[X]$ and $K[X, Y]$, respectively by a coloring $c$.

Next, we state a canonical Ramsey theorem which is essential for our proofs.

Theorem 3 (Deuber [7], Erdös-Rado [8]). For any integers $m, l, r$, there is a smallest integer $n=E R(m, l, r)$, such that any edge-coloring of $K_{n}$ contains either a monochromatic copy of $K_{m}$, a lexically colored copy of $K_{l}$, or a rainbow copy of $K_{r}$.

The number $E R$ is typically referred to as Erdős-Rado number, with best bound in the symmetric case provided by Lefmann and Rödl [15], in the following form: $2^{c_{1} \ell^{2}} \leqslant$ $E R(\ell, \ell, \ell) \leqslant 2^{c_{2} \ell^{2} \log \ell}$, for some constants $c_{1}, c_{2}$. 


\section{Proof of Theorem 2}

If $G$ is a graph with chromatic number at least 3 , then $\max R\left(n ; G, C_{k}\right)=n\left(\frac{k-2}{2}+\frac{1}{k-1}\right)+$ $O(1)$ as was proven in [3].

For the rest of the proof we shall assume that $G$ is a bipartite graph, not a star, with $s=s(G), t=t(G)$, and $G \subseteq K_{s, t}$. Note that $2 \leqslant s \leqslant t$. Let $K=K_{n}$. If $s \geqslant k$, then the lower bound on $\max R\left(n ; G, C_{k}\right)$ is given by $c^{*}$, a special $k$-anticyclic coloring. The upper bound follows from (1).

Suppose $s<k$. The lower bound is provided by a coloring $c^{* *}$. Since $\max R\left(n ; G, C_{k}\right) \leqslant$ $\max R\left(n ; K_{s, t}, C_{k}\right)$, in order to provide an upper bound on $\max R\left(n ; G, C_{k}\right)$, we shall be giving an upper bound on $\max R\left(n ; K_{s, t}, C_{k}\right)$.

The idea of the proof is as follows. We consider an edge coloring $c$ of $K=(V, E)$ with no monochromatic $K_{s, t}$ and no rainbow $C_{k}$, and estimate the number of colors in this coloring by analyzing specific vertex subsets: $L, A, B$, where $L$ is the vertex set of the largest weakly lexically colored complete subgraph, $A$ is the set of vertices in $V \backslash L$ which "disagrees" with coloring of $L$ on some edges incident to the initial part of $L$, and $B$ is the set of vertices in $V \backslash L$ which "disagrees" with coloring of $L$ on some edges incident to the terminal part of $L$. Let $V^{\prime}=V \backslash L$. We are counting the colors in the following order: first colors induced by $V^{\prime}$ which are not used on any edges incident to $L$ or any edges induced by $L$, then colors used on edges between $V^{\prime}$ and $L$ which are not induced by $L$, finally colors induced by $L$.

Now, we provide a formal proof. Assume that $n$ is sufficiently large such that $n \geqslant$ $E R(s+t, 3 s k+t+1, k)$. Let $c$ be a coloring of $E(K)$ with no monochromatic copy of $K_{s, t}$ and no rainbow copy of $C_{k}, c: E(K) \rightarrow \mathbb{N}$. Then there is a lexically colored copy of $K_{3 s k+t+1}$ by the canonical Ramsey theorem. Let $L$ be a vertex set of a largest weakly lexically colored $K_{q}, q \geqslant 3 s k+t+1$, say $L=\left\{x_{1}, \ldots, x_{q}\right\}$ and $c\left(x_{i} x_{j}\right)=\lambda\left(x_{i}\right)$ for $1 \leqslant i<j \leqslant q$, for some function $\lambda: L \rightarrow \mathbb{N}$. If $X=\left\{x_{i_{1}}, \ldots, x_{i_{\ell}}\right\} \subseteq L$ and $\lambda\left(x_{i_{1}}\right)=\cdots=\lambda\left(x_{i_{\ell}}\right)=j$ for some $j$, then we denote $\lambda(X)=j$. We write, for $i \leqslant j$, $x_{i} L x_{j}:=\left\{x_{i}, x_{i+1}, \ldots, x_{j}\right\}$, and for $i>j, x_{i} L x_{j}:=\left\{x_{i}, x_{i-1}, \ldots, x_{j}\right\}$. We say that $x_{i}$ precedes $x_{j}$ if $i<j$.

Let $T_{t}, T_{s k+t}, T_{2 s k+t}$, and $T_{3 s k+t}$ be the tails of $L$ of size $t, s k+t, 2 s k+t$, and $3 s k+t$ respectively, i.e.,

$$
\begin{aligned}
T_{t} & :=\left\{x_{q-t+1}, x_{q-t+2}, \ldots, x_{q}\right\} \\
T_{s k+t} & :=\left\{x_{q-s k-t+1}, x_{q-s k-t+2}, \ldots, x_{q}\right\} \\
T_{2 s k+t} & :=\left\{x_{q-2 s k-t+1}, x_{q-2 s k-t+2}, \ldots, x_{q}\right\}, \\
T_{3 s k+t} & :=\left\{x_{q-3 s k-t+1}, x_{q-3 s k-t+2}, \ldots, x_{q}\right\},
\end{aligned}
$$

see Figure 1. 


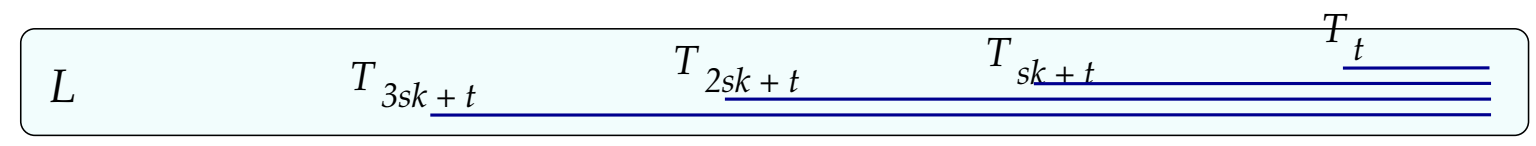

Figure 1: $T_{t}, T_{s k+t}, T_{2 s k+t}$, and $T_{3 s k+t}$

We shall use these tails to count the number of colors: the common difference, sk, of sizes of tails is from observations below(Claims $0.1-0.3$ ). The first tail $T_{t}$ is used in Claims $0.1-0.3$ and to find monochromatic copy of $K_{s, t}$. The third tail $T_{2 s k+t}$ is the main tool used in Part 1, 2 of the proof, it helps finding rainbow copy of $C_{k}$. The other tails $T_{s k+t}$ and $T_{3 s k+t}$ are for technical reasons used in Claim 2.1 and Claim 1.3, respectively. Note that the size of the fourth tail is used in the second parameter of Erdős-Rado number bounding $n$.

We start by splitting the vertices in $V \backslash L$ according to "agreement" or "disagreement" of a corresponding colors used in $L \backslash T_{2 s k+t}$ and in edges between $L$ and $V \backslash L$. Formally, let $V^{\prime}=V \backslash L$, and

$$
\begin{aligned}
A:= & \left\{v \in V^{\prime} \mid \text { there exists } y \in L \backslash T_{2 s k+t} \text { such that } c(v y) \neq \lambda(y)\right\} \\
B:= & \left\{v \in V^{\prime} \mid c(v x)=\lambda(x), x \in L \backslash T_{2 s k+t},\right. \\
& \text { and there exists } \left.y \in T_{2 s k+t} \backslash\left\{x_{q}\right\} \text { such that } c(v y) \neq \lambda(y)\right\} .
\end{aligned}
$$

Note that $V^{\prime}-A-B=\left\{v \in V^{\prime} \mid c(v x)=\lambda(x), x \in L \backslash\left\{x_{q}\right\}\right\}=\emptyset$ since otherwise $L$ is not the largest weakly colored complete subgraph. Thus

$$
V=L \cup A \cup B
$$

Let $c_{0}:=c(L) \cup c\left(V^{\prime}, L\right)$. In the first part of the proof we bound $\left|(c(B) \cup c(B, A)) \backslash c_{0}\right|+$ $|c(B, L) \backslash c(L)|$, in the second part we bound $\left|c(A) \backslash c_{0}\right|+|c(A, L) \backslash c(L)|+|c(L)|$.

Claim 0.1 Let $x \in L \backslash T_{t}$. Then $\left|\left\{y \in L \backslash T_{t} \mid \lambda(x)=\lambda(y)\right\}\right| \leqslant s-1<s$.

If this claim does not hold, the corresponding $y$ 's and $T_{t}$ induce a monochromatic $K_{s, t}$.

Claim 0.2 Let $y, y^{\prime} \in L \backslash T_{t}$ such that $\left|y L y^{\prime}\right|>(s-1) \ell+1$ for some $\ell \geqslant 0$. Then $\left|c\left(y L y^{\prime}\right)\right| \geqslant \ell+1$.

It follows from Claim 0.1 .

Claim 0.3 Let $v, v^{\prime} \in V^{\prime}$ and $y, y^{\prime} \in L \backslash T_{t}$ such that $y$ precedes $y^{\prime}$. Let $P$ be a rainbow path from $v$ to $v^{\prime}$ in $V^{\prime}$ with $1 \leqslant|V(P)| \leqslant k-2$ and colors not from $c_{0}$. If $c(v y) \neq \lambda(y)$, $c\left(v^{\prime} y^{\prime}\right) \notin\{c(v y), \lambda(y)\}$, and $\left|y L y^{\prime}\right|>(s-1)(k-|V(P)|)+1$, then there is a rainbow $C_{k}$ induced by $V(P) \cup y L y^{\prime}$.

Indeed, by Claim 0.2, $\left|c\left(y L y^{\prime}\right)\right| \geqslant k-|V(P)|+1$. Hence $\left|c\left(y L y^{\prime}\right) \backslash\left\{c(v y), c\left(v^{\prime} y^{\prime}\right)\right\}\right| \geqslant$ $k-|V(P)|-1$. So we can find a rainbow path on $k-|V(P)|$ vertices in $L$ with endpoints $y$ 


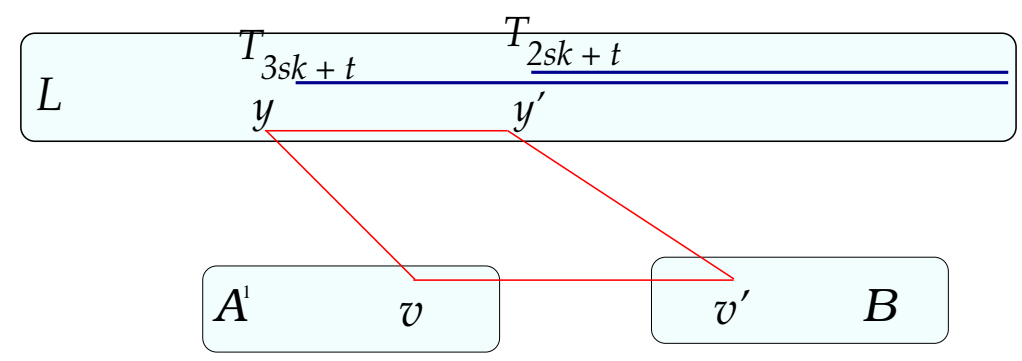

Figure 2: A rainbow $C_{k}$ in Claim 1.3

and $y^{\prime}$ of colors from $c\left(y L y^{\prime}\right) \backslash\left\{c(v y), c\left(v^{\prime} y^{\prime}\right)\right\}$, which together with $V(P)$ induce a rainbow $C_{k}$ since colors of $P$ are not from $c_{0}$.

\section{PART 1}

We shall show that $\left|(c(B) \cup c(B, A)) \backslash c_{0}\right|+|c(B, L) \backslash c(L)| \leqslant$ const $=$ const $(k, s, t)$.

Claim $1.1|B|<E R(s+t, 2 s k+t+1, k)$.

Suppose $|B| \geqslant E R(s+t, 2 s k+t+1, k)$. Then there is a lexically colored copy of a complete subgraph on a vertex set $Y \subseteq B$ of size $2 s k+t+1$. Then $(L \cup Y) \backslash T_{2 s k+t}$ is weakly lexical, which contradicts the maximality of $L$.

Claim 1.2 $|c(B, L) \backslash c(L)| \leqslant(2 s k+t)|B|$.

$|c(B, L) \backslash c(L)| \leqslant\left|c\left(B, T_{2 s k+t}\right)\right| \leqslant(2 s k+t)|B|$ by the definition of $B$.

Claim $1.3\left|(c(B) \cup c(B, A)) \backslash c_{0}\right|<\left(\begin{array}{c}E R(s+t, 3 s k+t+1, k) \\ 2\end{array}\right)$.

Let $A=A^{1} \cup A^{2}$, where $A^{1}:=\left\{v \in A \mid\right.$ there exists $y \in L \backslash T_{3 s k+t}$ with $\left.c(v y) \neq \lambda(y)\right\}$, and $A^{2}:=A \backslash A^{1}$.

First, we show that $c\left(B, A^{1}\right) \subseteq c_{0}$. Assume that $c\left(v^{\prime} v\right) \notin c_{0}$ for some $v \in A^{1}$ and $v^{\prime} \in B$ with $c(v y) \neq \lambda(y)$ for some $y \in L \backslash T_{3 s k+t}$ and $c\left(v^{\prime} x\right)=\lambda(x)$ for any $x \in L \backslash T_{2 s k+t}$. From Claim 0.1, we can find $y^{\prime}$, one of the last $2 s-1$ elements in $T_{3 s k+t} \backslash T_{2 s k+t}$ such that $\lambda\left(y^{\prime}\right)$ is neither $c(v y)$ nor $\lambda(y)$. Since $\lambda\left(y^{\prime}\right)=c\left(v^{\prime} y^{\prime}\right)$, we have that $c\left(v^{\prime} y^{\prime}\right) \notin\{c(v y), \lambda(y)\}$. Moreover we have $\left|y L y^{\prime}\right|>(s-1)(k-2)+1$. By Claim 0.3, there is a rainbow $C_{k}$ induced by $\left\{v, v^{\prime}\right\} \cup y L y^{\prime}$, see Figure 2 .

Second, we shall observe that $\left|A^{2} \cup B\right|<E R(s+t, 3 s k+t+1, k)$ by the argument similar to one used in Claim 1.1. We see that otherwise $A^{2} \cup B$ contains a lexically colored complete subgraph on $3 s k+t+1$ vertices, which together with $L-T_{3 s k+t}$ gives a larger than $L$ weakly lexically colored complete subgraph. 


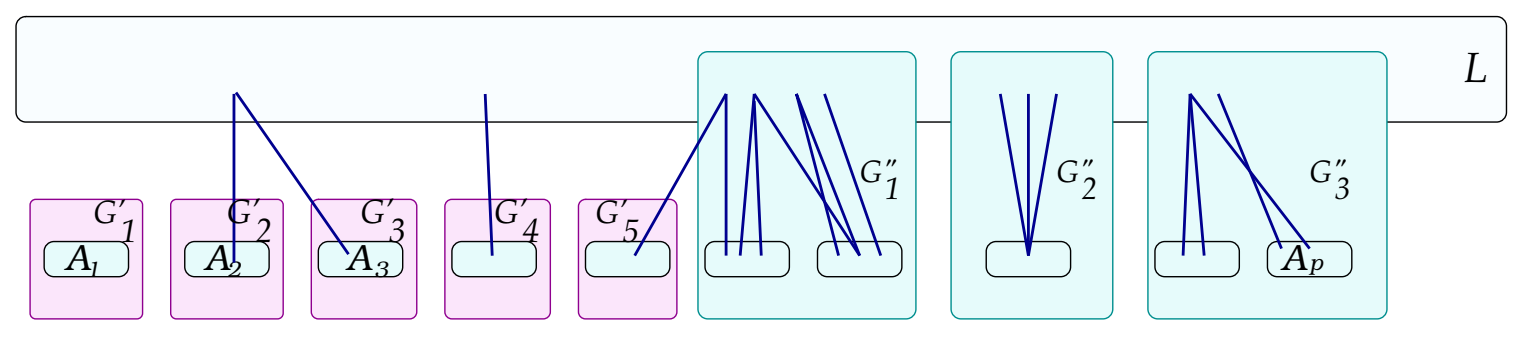

Figure 3: $G_{1}$ and $G_{2}$

\section{PART 2}

We shall show that $\left|c(A) \backslash c_{0}\right|+|c(A, L) \backslash c(L)|+|c(L)| \leqslant n\left(\frac{s-2}{2}+\frac{1}{s-1}\right)$.

In order to count the number of colors in $A$ and $(A, L)$, we consider a representing graph of these colors as follows. First, consider a set $E^{\prime}$ of edges from $K[A]$ having exactly one edge of each color from $c(A) \backslash c_{0}$. Second, consider a set of edges $E^{\prime \prime}$ from the bipartite graph $K[A, L]$ having exactly one edge of each color from $c(A, L) \backslash c(L)$. Let $G$ be a graph with edge-set $E^{\prime} \cup E^{\prime \prime}$ spanning $A$. Then $\left|c(A) \backslash c_{0}\right|+|c(A, L) \backslash c(L)|=|E(G)|$.

We need to estimate the number of edges in $G$. Let $A_{1}, \ldots, A_{p}$ be sets of vertices of the connected components of $G[A]$. Let $L_{1}, \ldots, L_{p}$ be sets of the neighbors of $A_{1}, \ldots, A_{p}$ in $L$ respectively, i.e., for $1 \leqslant i \leqslant p, L_{i}:=\left\{x \in L \mid\{x, y\} \in E(G)\right.$ for some $\left.y \in A_{i}\right\}$. Let

$$
\begin{aligned}
G_{1} & :=\bigcup_{i:\left|E\left(G\left[A_{i}, L_{i}\right]\right)\right| \leqslant 1} G\left[A_{i}\right], \\
G_{2} & :=\bigcup_{i:\left|E\left(G\left[A_{i}, L_{i}\right]\right)\right| \geqslant 2} G\left[A_{i} \cup L_{i}\right] .
\end{aligned}
$$

Let $G_{1}^{\prime}, \ldots, G_{p_{1}}^{\prime}$ be the connected components of $G_{1}$, and let $G_{1}^{\prime \prime}, \ldots, G_{p_{2}}^{\prime \prime}$ be the connected components of $G_{2}$. See Figure 3 for an example of $G_{1}$ and $G_{2}$.

Claim 2.1 We may assume that $V(G) \cap L \subseteq L \backslash T_{s k+t}$.

For a fixed $v \in A$, let $\omega$ be a color in $c(v, L) \backslash c(L)$, if such exists. Let $L(\omega):=$ $\{x \in L \mid c(v x)=\omega\}$. Suppose $L(\omega) \subseteq T_{s k+t}$. Since $v \in A$, there exists $y \in L \backslash T_{2 s k+t}$ such that $c(v y) \neq \lambda(y)$. Let $y^{\prime} \in L(\omega) \subseteq T_{s k+t}$. Then $c\left(v y^{\prime}\right) \notin\{c(v y), \lambda(y)\}$. Since $\left|y L y^{\prime}\right|>(s-1) k+1>(s-1)(k-1)+1$, there is a rainbow $C_{k}$ induced by $\{v\} \cup y L y^{\prime}$ by Claim 0.3, see figure 4. Therefore $L(\omega) \cap\left(L \backslash T_{s k+t}\right) \neq \emptyset$. Hence we can choose edges for the edge set $E^{\prime \prime}$ of $G$ only from $K\left[A, L \backslash T_{s k+t}\right]$.

Claim 2.2 For every $i, 1 \leqslant i \leqslant p, K\left[A_{i}, T_{t}\right]$ is monochromatic; for every $j, 1 \leqslant j \leqslant p_{2}$, $K\left[V\left(G_{j}^{\prime \prime}\right), T_{t}\right]$ is monochromatic. In particular, for every $h, 1 \leqslant h \leqslant p_{1}, K\left[V\left(G_{i}^{\prime}\right), T_{t}\right]$ is monochromatic.

1. Fix $i, 1 \leqslant i \leqslant p$. We show that $K\left[A_{i}, T_{t}\right]$ is monochromatic. Let $v \in A_{i}$ and $y \in L \backslash T_{2 s k+t}$ with $c(v y) \neq \lambda(y)$. 


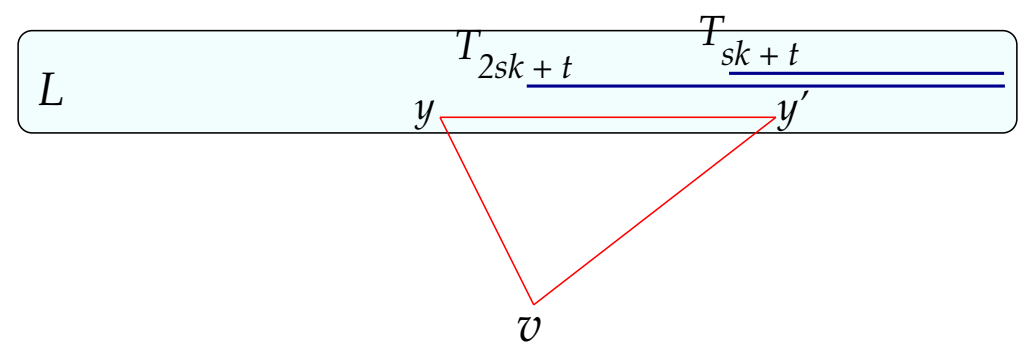

Figure 4: A rainbow $C_{k}$ in Claim 2.1 and Claim 2.2-1.(1)

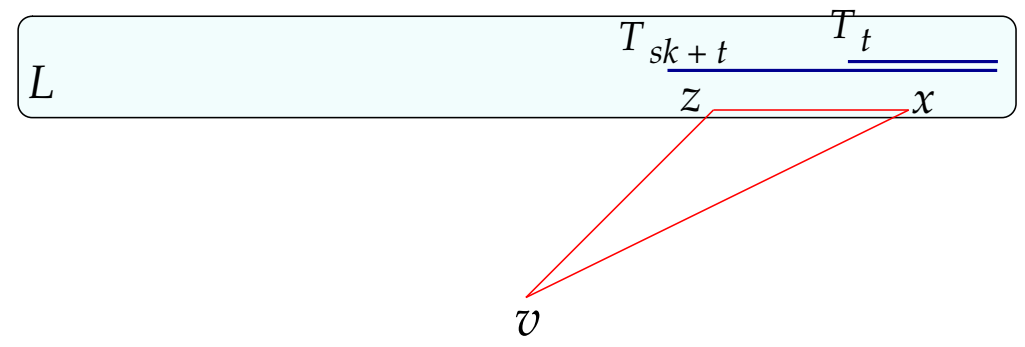

Figure 5: A rainbow $C_{k}$ in Claim 2.2-1.(2)

(1) For any $y^{\prime} \in T_{s k+t}, c\left(v y^{\prime}\right)$ is either $c(v y)$ or $\lambda(y)$. Indeed if $c\left(v y^{\prime}\right) \notin\{c(v y), \lambda(y)\}$, then there is a rainbow $C_{k}$ induced by $\{v\} \cup y L y^{\prime}$ by Claim 0.3 , see Figure 4 .

(2) $\left|c\left(v, T_{t}\right)\right|=1$. Indeed, let $L^{y}=\left\{x \in T_{s k+t} \backslash T_{t} \mid \lambda(x) \neq c(v y)\right.$ and $\left.\lambda(x) \neq \lambda(y)\right\}$. Then by Claim 0.1, $\left|L^{y}\right| \geqslant\left|T_{s k+t} \backslash T_{t}\right|-2(s-1)+1>(s-1)(k-3)+1$. Hence $\left|c\left(L^{y}\right)\right| \geqslant k-2$ by Claim 0.2 . Let $z$ be the vertex in $L^{y}$ preceding every other vertex in $L^{y}$. Suppose there is $x \in T_{t}$ such that $c(v x) \neq c(v z)$. Since $c\left(L^{y}\right) \subseteq c(z L x)$, there exists a rainbow path from $z$ to $x$ on $k-1$ vertices in $T_{s k+t}$ of colors disjoint from $\{c(v y), \lambda(y)\}$. So there is a rainbow $C_{k}$ induced by $\{v\} \cup z L x$, see Figure 5 . Therefore for any $x \in T_{t}, c(v x)=c(v z) \in\{c(v y), \lambda(y)\}$.

(3) For any neighbor $v^{\prime}$ of $v$ in $G\left[A_{i}\right]$, if such exists, $c\left(v^{\prime}, T_{t}\right)=c\left(v, T_{t}\right)$. Indeed, we see that for any $y^{\prime} \in T_{s k+t}, c\left(v^{\prime} y^{\prime}\right) \in\{c(v y), \lambda(y)\}$, otherwise there is a rainbow $C_{k}$ induced by $\left\{v, v^{\prime}\right\} \cup y L y^{\prime}$ by Claim 0.3. Also we see that for any $x \in T_{t}$, $c\left(v^{\prime} x\right)=c(v z) \in\{c(v y), \lambda(y)\}$, where $z$ is defined above; otherwise there is a rainbow $C_{k}$ induced by $\left\{v, v^{\prime}\right\} \cup z L x$, see Figure 6. Therefore $c\left(v^{\prime}, T_{t}\right)=c\left(v, T_{t}\right)$.

(4) Since $G\left[A_{i}\right]$ is connected, $K\left[A_{i}, T_{t}\right]$ is monochromatic of color $c(v z)$.

Note that to avoid a monochromatic $K_{s, t}$, we must have that $\left|A_{i}\right| \leqslant s-1 \leqslant k-2$ for $1 \leqslant i \leqslant p$.

2. Fix $j, 1 \leqslant j \leqslant p_{2}$. We show that $K\left[V\left(G_{j}^{\prime \prime}\right), T_{t}\right]$ is monochromatic. 


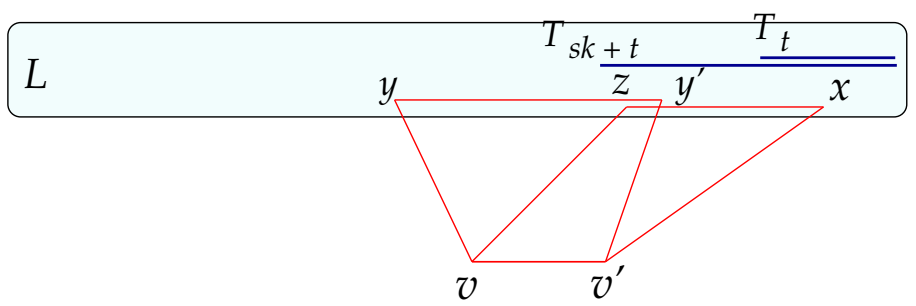

Figure 6: Rainbow $C_{k}$ 's in Claim 2.2-1.(3)

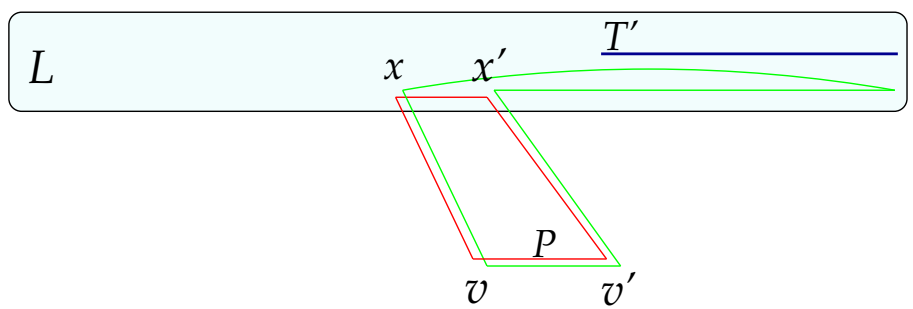

Figure 7: Rainbow $C_{k}$ 's in Claim 2.2-2.(1): red when $|P|=k-2$, green when $|P|<k-2$.

(1) $K\left[V\left(G_{j}^{\prime \prime}\right) \cap L, T_{t}\right]$ is monochromatic. Indeed, since $G_{j}^{\prime \prime}$, a connected component of $G$, is a union of $G\left[A_{i} \cup L_{i}\right]$ 's satisfying $\left|E\left(G\left[A_{i}, L_{i}\right]\right)\right| \geqslant 2$, by the connectivity, it is enough to show that $\lambda(x)=\lambda\left(x^{\prime}\right)$ for any $x, x^{\prime} \in L_{i}$ for $L_{i}$ in $G_{j}^{\prime \prime}$, where $x$ precedes $x^{\prime}$. From Claim 2.1, we may assume that $x, x^{\prime}$ are in $L \backslash T_{s k+t}$. Suppose $\lambda(x) \neq \lambda\left(x^{\prime}\right)$. Let $v, v^{\prime} \in A_{i}$ such that $\{v, x\}$ and $\left\{v^{\prime}, x^{\prime}\right\}$ are edges of $G$ (possibly $v=v^{\prime}$ ). Let $P$ denote a set of vertices on a path from $v$ to $v^{\prime}$ in $G\left[A_{i}\right]$. Then $1 \leqslant|P| \leqslant k-2$ since $\left|A_{i}\right| \leqslant k-2$. If $|P|=k-2$, then $P \cup\left\{x, x^{\prime}\right\}$ induces a rainbow $C_{k}$, otherwise so does $P \cup\{x\} \cup x^{\prime} L x_{q}$ from Claim 0.3, see Figure 7. Therefore $\lambda(x)=\lambda\left(x^{\prime}\right)$.

(2) $K\left[V\left(G_{j}^{\prime \prime}\right), T_{t}\right]$ is monochromatic. To prove this, consider $i$ such that $G\left[A_{i}, L_{i}\right] \subseteq G_{j}^{\prime \prime}$. Observe first that $K\left[A_{i}, T_{t}\right]$ and $K\left[L_{i}, T_{t}\right]$ are monochromatic by 1.(4) and 2.(1). Next, we shall show that $c\left(A_{i}, T_{t}\right)=\lambda\left(L_{i}\right)$. Suppose $c\left(A_{i}, T_{t}\right) \neq \lambda\left(L_{i}\right)$ for some $i$ such that $G\left[A_{i} \cup L_{i}\right] \subseteq G_{j}^{\prime \prime}$. Let $v, v^{\prime} \in A_{i}$ and $x, x^{\prime} \in L_{i}$ such that $\{v, x\}$ and $\left\{v^{\prime}, x^{\prime}\right\}$ are edges of $G$ (possibly either $v=v^{\prime}$ or $x=x^{\prime}$ ). Since $\left|E\left(G\left[A_{i}, L_{i}\right]\right)\right| \geqslant 2$, we can find such vertices. So $c(v x) \neq c\left(v^{\prime} x^{\prime}\right)$ and $\left\{c(v x), c\left(v^{\prime} x^{\prime}\right)\right\} \cap c(L)=\emptyset$. We may assume that $x, x^{\prime} \in L \backslash T_{s k+t}$ by Claim 2.1. Since $c\left(A_{i}, T_{t}\right) \neq \lambda\left(L_{i}\right), c(v x)=c\left(v^{\prime} x^{\prime}\right)=$ $c\left(A_{i}, T_{t}\right)$, otherwise there is a rainbow $C_{k}$ induced by $\{v\} \cup x L x_{q}$ or $\left\{v^{\prime}\right\} \cup x^{\prime} L x_{q}$ by Claim 0.3, see Figure 8. Then it contradicts the fact that $c(v x) \neq c\left(v^{\prime} x^{\prime}\right)$.

We have that for any $i$ such that $G\left[A_{i}, L_{i}\right] \subseteq G_{j}^{\prime \prime}, c\left(A_{i}, T_{t}\right)=\lambda\left(L_{i}\right)$. This implies that $K\left[A_{i} \cup L_{i}, T_{t}\right]$ is monochromatic of color $\lambda\left(L_{i}\right)$. Since $G_{j}^{\prime \prime}$ is connected and $A_{i} \mathrm{~s}$ are disjoint, we have that for any $i, i^{\prime}$ such that $G\left[A_{i}, L_{i}\right], G\left[A_{i^{\prime}}, L_{i^{\prime}}\right] \subseteq G_{j}^{\prime \prime}, L_{i} \cap L_{i^{\prime}} \neq \emptyset$, so $\lambda\left(L_{i}\right)=\lambda\left(L_{i^{\prime}}\right)=\lambda$, for some $\lambda$. Therefore $K\left[V\left(G_{j}^{\prime \prime}\right), T_{t}\right]$ is monochromatic of color $\lambda$. 


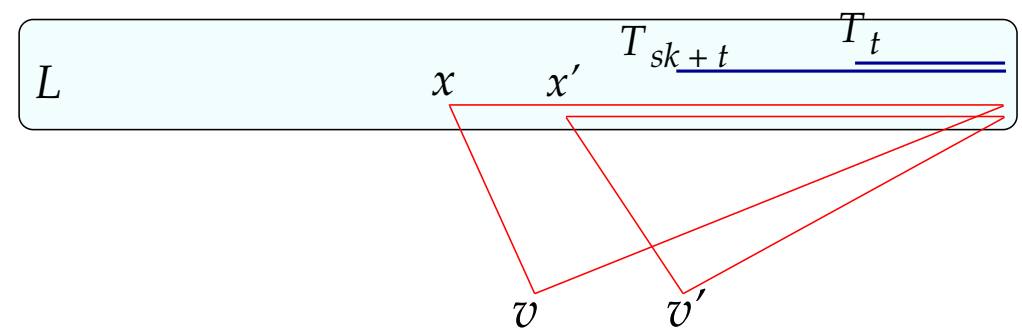

Figure 8: Rainbow $C_{k}$ 's for Claim 2.2-2.(2).

Claim 2.3 For $1 \leqslant i \leqslant p_{1}$ and $1 \leqslant j \leqslant p_{2}, 1 \leqslant\left|V\left(G_{i}^{\prime}\right)\right| \leqslant s-1$ and $1 \leqslant\left|V\left(G_{j}^{\prime \prime}\right)\right| \leqslant s-1$.

This claim now follows from the previous instantly.

The following claim deals with a small quadratic optimization problem we shall need.

Claim 2.4 Let $n, s \in \mathbb{N}$. Suppose $n$ is sufficiently large and $s \geqslant 2$. Let $\xi_{1}, \ldots, \xi_{m} \in \mathbb{N}$, $1 \leqslant \xi_{i} \leqslant s-1$ and $\sum_{i=1}^{m} \xi_{i} \leqslant n$. Then

$$
\sum_{i=1}^{m}\left(\begin{array}{c}
\xi_{i}-1 \\
2
\end{array}\right) \leqslant n\left(\frac{s-4}{2}+\frac{1}{s-1}\right)
$$

The equality holds if and only if $m=\frac{n}{s-1}$ and $\xi_{1}=\cdots=\xi_{m}=s-1$.

See the appendix A for the proof.

Claim 2.5 $\left|c(A) \backslash c_{0}\right|+|c(A, L) \backslash c(L)|+|c(L)|=|E(G)|+|c(L)| \leqslant n\left(\frac{s-2}{2}+\frac{1}{s-1}\right)$.

We have that

$$
|E(G)| \leqslant\left(\left|E\left(G_{1}\right)\right|+p_{1}\right)+\left|E\left(G_{2}\right)\right|=\sum_{i=1}^{p_{1}}\left|E\left(G_{i}^{\prime}\right)\right|+p_{1}+\sum_{i=1}^{p_{2}}\left|E\left(G_{i}^{\prime \prime}\right)\right| .
$$

Moreover each component $G_{i}^{\prime \prime}$ of $G_{2}$ contributes at most 1 to $|c(L)|$ by Claim 2.2, and $G_{1}$ and $G_{2}$ are vertex disjoint. So

$$
|c(L)| \leqslant n-\left|V\left(G_{1}\right)\right|-\left|V\left(G_{2}\right)\right|+p_{2}=n-\sum_{i=1}^{p_{1}}\left|V\left(G_{i}^{\prime}\right)\right|-\sum_{i=1}^{p_{2}}\left|V\left(G_{i}^{\prime \prime}\right)\right|+p_{2}
$$


Hence we have

$$
\begin{aligned}
\left|c(A) \backslash c_{0}\right|+|c(A, L) \backslash c(L)|+|c(L)|=|E(G)|+|c(L)| \\
\leqslant \sum_{i=1}^{p_{1}}\left|E\left(G_{i}^{\prime}\right)\right|+p_{1}+\sum_{i=1}^{p_{2}}\left|E\left(G_{i}^{\prime \prime}\right)\right|+n-\sum_{i=1}^{p_{1}}\left|V\left(G_{i}^{\prime}\right)\right|-\sum_{i=1}^{p_{2}}\left|V\left(G_{i}^{\prime \prime}\right)\right|+p_{2} \\
\quad=\sum_{i=1}^{p_{1}}\left|E\left(G_{i}^{\prime}\right)\right|+\sum_{i=1}^{p_{2}}\left|E\left(G_{i}^{\prime \prime}\right)\right|-\sum_{i=1}^{p_{1}}\left(\left|V\left(G_{i}^{\prime}\right)\right|-1\right)-\sum_{i=1}^{p_{2}}\left(\left|V\left(G_{i}^{\prime \prime}\right)\right|-1\right)+n \\
\leqslant \sum_{i=1}^{p_{1}}\left(\begin{array}{c}
\left|V\left(G_{i}^{\prime}\right)\right| \\
2
\end{array}\right)+\sum_{i=1}^{p_{2}}\left(\begin{array}{c}
p_{2} \\
2
\end{array}\right)-\sum_{i=1}^{p_{1}}\left(\left|V\left(G_{i}^{\prime}\right)\right|-1\right)-\sum_{i=1}^{p_{1}}\left(\left|V\left(G_{i}^{\prime \prime}\right)\right|-1\right)+n \\
\quad=\sum_{i=1}^{p_{1}}\left(\begin{array}{c}
\left|V\left(G_{i}^{\prime}\right)\right|-1 \\
2
\end{array}\right)+\sum_{i=1}^{p_{2}}\left(\begin{array}{c}
\left|V\left(G_{i}^{\prime \prime}\right)\right|-1 \\
2
\end{array}\right)+n
\end{aligned}
$$

For $1 \leqslant i \leqslant p_{1}+p_{2}$, let

$$
\xi_{i}=\left\{\begin{array}{ll}
\left|V\left(G_{i}^{\prime}\right)\right|, & \text { if } 1 \leqslant i \leqslant p_{1} \\
\left|V\left(G_{i-p_{1}}^{\prime \prime}\right)\right|, & \text { if } p_{1}+1 \leqslant i \leqslant p_{1}+p_{2}
\end{array} .\right.
$$

Then $\sum_{i=1}^{p_{1}+p_{2}} \xi_{i} \leqslant n$ and $1 \leqslant \xi_{i} \leqslant s-1$ for $1 \leqslant i \leqslant p_{1}+p_{2}$ by Claim 2.3 .

From Claim 2.4, we get

$$
\begin{aligned}
& \left|c(A) \backslash c_{0}\right|+|c(A, L) \backslash c(L)|+|c(L)| \\
& \leqslant \sum_{i=1}^{p_{1}+p_{2}}\left(\begin{array}{c}
\xi_{i}-1 \\
2
\end{array}\right)+n \leqslant n\left(\frac{s-2}{2}+\frac{1}{s-1}\right) .
\end{aligned}
$$

This concludes Part 2 of the proof.

Combining Parts 1 and 2, we see that the total number of colors is at most

$$
\begin{aligned}
& \left|(c(B) \cup c(B, A)) \backslash c_{0}\right|+|c(B, L) \backslash c(L)|+\left|c(A) \backslash c_{0}\right|+|c(A, L) \backslash c(L)|+|c(L)| \\
& <\left(\begin{array}{c}
E R(s+t, 3 s k+t+1, k) \\
2
\end{array}\right)+(2 s k+t) E R(s+t, 2 s k+t+1, k)+n\left(\frac{s-2}{2}+\frac{1}{s-1}\right) \\
& \leqslant g+n\left(\frac{s-2}{2}+\frac{1}{s-1}\right),
\end{aligned}
$$

where $g=g(s, t, k)=E R^{2}(s+t, 3 s k+t+1, k)$. 


\section{More precise results for $C_{4}$}

For a coloring $c$ of $E\left(K_{n}\right)$ and a vertex $v$, let $N_{c}(v)$ be the set of colors between $v$ and $V\left(K_{n}\right) \backslash\{v\}$, not used on edges spanned by $V\left(K_{n}\right) \backslash\{v\}$. Let $n_{c}(v)=\left|N_{c}(v)\right|$. Note that $c(u v) \in N_{c}(u) \cap N_{c}(v)$ if and only if the color $c(u v)$ is used only on the edge $u v$ in the coloring $c$. We call this color a unique color in $c$. For a path $P=v_{1} v_{2} \cdots v_{k}$, we say that the path $P$ is good if $c\left(v_{i} v_{i+1}\right) \in N_{c}\left(v_{i}\right)$ for $i=1, \ldots, k-1$.

Lemma 1. Let $c$ be an edge-coloring of $K_{n}$ with no rainbow $C_{k}$. If for all $v \in V\left(K_{n}\right)$, $n_{c}(v) \geqslant k-2$, then $(k-1) \mid n$ and $c$ is $k$-anticyclic.

Proof. Let $c$ be an edge-coloring of $K_{n}$ with no rainbow $C_{k}$. Suppose for all $v \in V\left(K_{n}\right)$, $n_{c}(v) \geqslant k-2$. Then for any $v \in V$, we can find a good path of length $k-2$ starting at $v$ by a greedy algorithm. Let this path be $v_{1} v_{2} \cdots v_{k-1}$, and let $c\left(v_{i} v_{i+1}\right)=i$ for $i=1, \ldots, k-2$. Let $V_{0}=\left\{v_{1}, \ldots, v_{k-1}\right\}$.

Claim 1 For any $u \in V \backslash V_{0}, c\left(u v_{1}\right)=1$ or $c\left(u v_{1}\right) \notin N_{c}\left(v_{1}\right)$.

Assume that $c\left(u v_{1}\right) \in N_{c}\left(v_{1}\right)$. If $c\left(u v_{1}\right) \neq 1$ then $c\left(u v_{k-1}\right)$ must be the same as $c\left(u v_{1}\right)$, otherwise $v_{1} \cdots v_{k-1} u v_{1}$ is a rainbow $C_{k}$. Thus, if $c\left(u v_{1}\right) \neq 1$ then $c\left(u v_{1}\right) \notin N_{c}\left(v_{1}\right)$.

Claim $2\left\{c\left(v_{1} v_{i}\right) \mid i=2, \ldots, k-1\right\}$ is a set of distinct colors from $N_{c}\left(v_{1}\right)$ and $n_{c}\left(v_{1}\right)=$ $k-2$.

From Claim 1 we see that the colors from $N_{c}\left(v_{1}\right)$ not equal to 1 appear only on edges $v_{1} v_{i}$ for $i=2, \ldots, k-1$. Since $n_{c}\left(v_{1}\right) \geqslant k-2$, all these edges have distinct colors from $N_{c}\left(v_{1}\right)$ and $n_{c}\left(v_{1}\right)=k-2$.

Claim 3 For any $u \in V \backslash V_{0}, c\left(u v_{k-1}\right) \notin N_{c}\left(v_{k-1}\right)$.

Assume otherwise, then $v_{2} v_{3} \cdots v_{k-1} u$ is a good path. Then $v_{1} v_{3} v_{4} \cdots v_{k-1} u v_{2} v_{1}$ is a rainbow $C_{k}$ from Claim 2.

Claim $4\left\{c\left(v_{i} v_{k-1}\right) \mid i=1, \ldots, k-2\right\}$ is a set of distinct colors from $N_{c}\left(v_{k-1}\right)$ and $n_{c}\left(v_{k-1}\right)=k-2$.

By Claim 3, we see that all edges of colors from $N_{c}\left(v_{k-1}\right)$ must occur on edges from $\left\{v_{i} v_{k-1}: i=1, \ldots, k-2\right\}$. Since $n_{c}\left(v_{k-1}\right) \geqslant k-2$, edges $v_{i} v_{k-1}, i=1, \ldots, k-2$ have distinct colors from $N_{c}\left(v_{k-1}\right)$ and $n_{c}\left(v_{k-1}\right)=k-2$.

Claim $5 V_{0}$ induces a rainbow complete subgraph with all colors unique in $c$. Moreover, for each $v_{i}$ and each $u \notin V_{0}, c\left(u v_{i}\right)$ is not unique in $c$.

This follows from the above claims since for $i=1, \ldots, k-1, v_{i} v_{i+1} \cdots v_{k-1} v_{1} v_{2} \cdots v_{i-1}$ is a good path, and $n_{c}\left(v_{i}\right)=k-2$.

Consider $u \notin V_{0}$ and a good path of length $k-2$ starting at $u$. Let the vertex set of this path be $V_{1}$. If $V_{0}$ and $V_{1}$ share a vertex, say $v_{i}$, then $v_{i} u$ has a unique color, a contradiction to Claim 5. Thus the graph is vertex-partitioned into copies of $K_{k-1}$ each rainbow colored with unique colors. To avoid a rainbow $C_{k}$, any edges between two fixed parts must have the same color. Therefore $(k-1) \mid n$ and $c$ is $k$-anticyclic. 
By induction on $n$ and the above lemma with $k=4$, we have the following results.

Corollary 4. $A R\left(n, C_{4}\right)=\left|c^{*}\right|=4 / 3 n+O(1)$.

Proof. We need to show that for any edge-coloring $c$ of $K_{n}$ with no rainbow $C_{4},|c| \leqslant$ $\left|c^{*}\right|=4 / 3 n+O(1)$.

We use induction on $n$. The statement trivially holds for $n=3$. Let $c$ be a coloring of $E\left[K_{n}\right]$ with no rainbow $C_{4}, n \geqslant 4$. If for all $v \in V\left(K_{n}\right), n_{c}(v) \geqslant 2$, then by Lemma $1, c$ is 4 -anticyclic. So $|c| \leqslant\left|c^{*}\right|$. Suppose there is a $v \in V\left(K_{n}\right)$ with $n_{c}(v) \leqslant 1$. Let $G=K_{n}-v$. Let $c^{\prime}$ be the coloring of $E(G)$ induced by $c$. Then by induction hypothesis, $\left|c^{\prime}\right| \leqslant 4 / 3(n-1)+O(1)$. Hence $|c| \leqslant\left|c^{\prime}\right|+1 \leqslant 4 / 3 n+O(1)$.

Theorem 5. Let $n \geqslant 3$. Let $G$ be a graph whose edges do not induce a star. Let $s=s(G)$ and $t=t(G)$ if $G$ is bipartite.

$$
\max R\left(n ; G, C_{4}\right)= \begin{cases}\frac{4}{3} n+O(1), & \text { if }(\chi(G)=2 \text { and } s(G) \geqslant 4) \text { or }(\chi(G) \geqslant 3) \\ n, & \text { otherwise }\end{cases}
$$

Proof. Suppose $(\chi(G)=2$ and $s(G) \geqslant 4)$ or $(\chi(G) \geqslant 3)$. For the lower bound, consider the 4-anticyclic coloring $c^{*}$. Each color class of $c^{*}$ is either $K_{1, m}, K_{2, m}$, or $K_{3, m}$ for some $m \geqslant 1$, thus $c^{*}$ contains no monochromatic copy of $G$. The upper bound follows from Corollary 4.

Suppose $G$ is bipartite and $s(G) \leqslant 3$. We use induction on $n$. The statement trivially holds for $n=3$. Let $c$ be a coloring of $E\left(K_{n}\right)$ with no monochromatic $G$ and no rainbow $C_{4}$. If $n_{c}(v) \geqslant 2$ for all $v \in V$, by Lemma 1 there is a color class of $c$ that induces a $K_{3,3 m}$ for some $m \geqslant 1$, which contains $G$. Hence we can find a $v \in V$ with $n_{c}(v) \leqslant 1$. Then by the induction hypothesis, $\max R\left(n ; G, C_{4}\right) \leqslant n$. The lower bound is obtained from the coloring $c^{* *}$ with $s=s(G)$ and $k=4$. Each color class of $c^{* *}$ is $K_{1, m}$ if $s(G)=2$, either $K_{1, m}$ or $K_{2, m}$ if $s(G)=3$ for some $m \geqslant 1$, thus $c^{* *}$ contains no monochromatic copy of $G$. The total number of colors in either cases is $n$. 


\section{A Proof of Claim 2.4}

Claim 2.4 Let $n, s \in \mathbb{N}$. Suppose $n$ is sufficiently large and $s \geqslant 2$. Let $\xi_{1}, \ldots, \xi_{m} \in \mathbb{N}$, $1 \leqslant \xi_{i} \leqslant s-1$ and $\sum_{i=1}^{m} \xi_{i} \leqslant n$. Then

$$
\sum_{i=1}^{m}\left(\begin{array}{c}
\xi_{i}-1 \\
2
\end{array}\right) \leqslant n\left(\frac{s-4}{2}+\frac{1}{s-1}\right)
$$

The equality holds if and only if $m=\frac{n}{s-1}$ and $\xi_{1}=\cdots=\xi_{m}=s-1$.

We use induction on $m$. If $m=1$, then

$$
\frac{(\xi-1)(\xi-2)}{2} \leqslant \frac{(s-2)(s-3)}{2} \leqslant n\left(\frac{s-4}{2}+\frac{1}{s-1}\right), \text { for any } n \geqslant s-1,
$$

where the first inequality becomes equality iff $\xi=s-1$, and the second does iff $n=s-1$. Suppose $m \geqslant 2, \sum_{i=1}^{m} \xi_{i} \leqslant n$, and $1 \leqslant \xi_{i} \leqslant s-1$ for $1 \leqslant i \leqslant m$. Since $\sum_{i=1}^{m-1} \xi_{i} \leqslant n-\xi_{m}$, by induction,

$$
\sum_{i=1}^{m-1}\left(\begin{array}{c}
\xi_{i}-1 \\
2
\end{array}\right) \leqslant\left(n-\xi_{m}\right)\left(\frac{s-4}{2}+\frac{1}{s-1}\right) \text {, for any } n \geqslant(m-1)(s-1)+\xi_{m}
$$

where the equality holds iff $m-1=\frac{n-\xi_{m}}{s-1}$ and $\xi_{1}=\cdots=\xi_{m-1}=s-1$. Hence it is enough to show that $\left(n-\xi_{m}\right)\left(\frac{s-4}{2}+\frac{1}{s-1}\right)+\left(\begin{array}{c}\xi_{m}-1 \\ 2\end{array}\right) \leqslant n\left(\frac{s-4}{2}+\frac{1}{s-1}\right)$ or equivalently $\xi_{m}\left(\frac{s-4}{2}+\frac{1}{s-1}\right)-\left(\begin{array}{c}\xi_{m}-1 \\ 2\end{array}\right) \geqslant 0$, and the equality holds iff $\xi_{m}=s-1$. If $\xi_{m}=1$, that is obvious. Assume $\xi_{m}>1$, then

$$
\begin{aligned}
& \xi_{m}\left(\frac{s-4}{2}+\frac{1}{s-1}\right)-\left(\begin{array}{c}
\xi_{m}-1 \\
2
\end{array}\right)=\xi_{m} \frac{(s-2)(s-3)}{2(s-1)}-\frac{\left(\xi_{m}-1\right)\left(\xi_{m}-2\right)}{2} \\
& =\frac{1}{2}\left(-\xi_{m}^{2}+\left(s-1+\frac{2}{s-1}\right) \xi_{m}-2\right)=\frac{1}{2}\left(-\xi_{m}+\frac{2}{s-1}\right)\left(\xi_{m}-(s-1)\right) \geqslant 0
\end{aligned}
$$

since $2 \leqslant \xi_{m} \leqslant s-1$.

Acknowledgments The authors thank the referee for a very careful reading and useful comments improving the presentation of the results. 


\section{References}

[1] B. Alexeev, On lengths of rainbow cycles, Electron. J. Combin. 13 (2006), Research Paper 105, 14 pp. (electronic).

[2] M. Axenovich, A. Kündgen, On a generalized anti-Ramsey problem, Combinatorica 21 (2001), no. 3, 335-349.

[3] M. Axenovich, P. Iverson, Edge-colorings avoiding rainbow and monochromatic subgraphs, Discrete Math., 2008, 308(20), 4710-4723.

[4] L. Babai, An anti-Ramsey theorem, Graphs Combin. 1 (1985), no.1, 23-28.

[5] P. Balister, A. Gyárfás, J. Lehel, R. Schelp, Mono-multi bipartite Ramsey numbers, designs, and matrices, Journal of Combinatorial Theory, Series A 113 (2006), 101112.

[6] B. Bollobás, Extremal Graph Theory, Academic Press, New York, 1978.

[7] W. Deuber, Canonization, Combinatorics, Paul Erdős is eighty, Vol. 1, 107-123, Bolyai Soc. Math. Stud., János Bolyai Math. Soc., Budapest, 1993.

[8] P. Erdős, R. Rado, A combinatorial theorem, J. London Math. Soc. 25, (1950), 249255.

[9] P. Erdős, M. Simonovits, V. T. Sós, Anti-Ramsey theorems, Infinite and finite sets (Colloq., Keszthely, 1973; dedicated to P. Erdős on his 60th birthday), Vol. II, pp. 633-643. Colloq. Math. Soc. Janos Bolyai, Vol. 10, North-Holland, Amsterdam, 1975.

[10] L. Eroh, O. R. Oellermann, Bipartite rainbow Ramsey numbers, Discrete Math. 277 (2004), 57-72.

[11] J. Fox, B. Sudakov, Ramsey-type problem for an almost monochromatic $K_{4}$, SIAM J. of Discrete Math. 23, (2008), 155-162.

[12] V. Jungic, T. Kaiser, D. Kral, A note on edge-colourings avoiding rainbow $K_{4}$ and monochromatic $K_{m}$, Electron. J. Combin. 16 (2009), no. 1, Note 19, 9 pp.

[13] A. Kostochka, D. Mubayi, When is an almost monochromatic $K_{4}$ guaranteed?, Combinatorics, Probability and Computing 17, (2008), no. 6, 823-830.

[14] R. Jamison, D. West, On pattern Ramsey numbers of graphs, Graphs Combin. 20 (2004), no. 3, 333-339.

[15] H. Lefmann, V. Rödl, On Erdős-Rado numbers, Combinatorica 15 (1995), 85-104.

[16] J. J. Montellano-Ballesteros, V. Neumann-Lara, An anti-Ramsey theorem on cycles, Graphs Combin. 21 (2005), no. 3, 343-354.

[17] J. J. Montellano-Ballesteros, V. Neumann-Lara, An anti-Ramsey theorem, Combinatorica 22 (2002), no. 3, 445-449.

[18] D. West, Introduction to graph theory, Prentice Hall, Inc., Upper Saddle River, NJ, 1996. xvi+512 pp. 